\title{
Propiedades psicométricas de la satisfaction with life scale en jóvenes angoleños: análisis factorial confirmatorio y modelo de respuesta graduada
}

\author{
Psychometric properties of satisfaction with life scale in angolan \\ youth: confirmatory factor analysis and graduate response model
}

\author{
Gutiérrez, M. ${ }^{1}$ Ph.D, Tomás, J.M. ${ }^{2}$ Ph.D, Jover, A. ${ }^{1}$ Psicol. \\ Universitat de Valéncia, España. ${ }^{1}$ Departamento de Psicología Evolutiva y de la Educación. ${ }^{2}$ Departamento \\ de Metodología de las Ciencias del Comportamiento. Correspondencia: tomasjm@uv.es
}

\section{RESUMEN}

La cantidad de investigaciones sobre bienestar subjetivo ha experimentado un aumento durante las últimas décadas. Por lo tanto, supone un gran interés disponer de instrumentos para su medición validados en los contextos de uso, para así aumentar la calidad de esta. El instrumento que más se emplea para medir la satisfacción con la vida es la "Satisfaction With Life Scale" (SWLS). Por ello, el propósito de este trabajo ha sido analizar las propiedades psicométricas de esta escala. La muestra la conformaron 1332 jóvenes angoleños, con edades comprendidas entre los 18 y los 30 años, procedentes de distintas localidades del norte, centro y sur de Angola. Los análisis de validación factorial confirman que los 5 ítems que configuran la escala conforman una estructura unifactorial. Los índices de consistencia interna, al igual que la validez nomológica, indican de nuevo que la SWLS es una escala válida y fiable para evaluar la satisfacción con la vida. Por último, los análisis de Teoría de respuesta del ítem ofrecen valiosa información sobre la fiabilidad de la medida, y sobre el carácter altamente discriminatorio de los ítems que la componen.

Palabras clave: Satisfacción con la vida; fiabilidad; validez; teoría de respuesta al ítem; autoestima; resiliencia. 
Gutiérrez et al - Propiedades psicométricas de la satisfaction with life scale en jóvenes angoleños

\section{ABSTRACT}

Research about subjective well-being has had an increase during the last decades. Therefore, it is of great interest to have validated measurement instruments in the contexts of use, in order to increase the quality of this. The most used instrument to measure life satisfaction is the "Satisfaction with Life Scale" (SWLS). Thus, the purpose of the present study has been to analyze the psychometric properties of this scale for its use in Angolan young adults. 1,332 people formed the sample, with ages varying from 18 to 30 years, coming from different northern, center and southern Angolan localities. The factorial validation analyzes confirm that the five (5) items that make up the scale conform a unifactorial structure. Internal consistency indexes, as well as nomological validity, again indicate that SWLS is a valid and reliable scale for assessing satisfaction with life. Finally, the item response theory analyzes provide valuable information on the reliability of the measure and on the highly discriminatory nature of the items that compose it. Finally, the theory analysis of the item response provide valuable information on the reliability of the measure and on the discriminatory nature of the items that compose it.

Key words: Satisfaction with life, Reliability, Validity, Item Response Theory, Selfesteem, Resilience.

\section{INTRODUCCIÓN}

El bienestar subjetivo supone un objeto de estudio de gran interés, ya que se usa frecuentemente en distintas áreas de las ciencias sociales y de la salud. Esto ha tenido como consecuencia que durante las últimas décadas se haya desarrollado abundante investigación sobre este tópico (Böckerman, Johanson, \& Saarni, 2012; Diener 1984; Diener, Oishi, \& Lucas, 2003; Eckersley, 2013; MonkTurner, \& Turner, 2012; Oswald \& Wu, 2010; Pavot \& Diener 1993; Renshaw, Long, \& Cook, 2015).

El término "bienestar" es conocido de un modo más general como "calidad de vida", la cual depende de la presencia de aquello que resulta satisfactorio acompañado de la evitación de todo aquello que hace sentir más desgraciada a la gente (Andrews, 1974). Si a esto le añadimos el factor subjetivo, se puede definir el bienestar subjetivo como todo aquello que haga sentir bien a una persona en relación con sus propios valores (Diener et al., 2003). Es un constructo que está compuesto, según Diener y Emmons (1984), por dos componentes, uno afectivo y otro cognitivo. Este último es denominado por Andrews y Withey (1976) como satisfacción con la vida. Estos componentes muestran diferencias entre sí (Beiser, 1974; DeHaes, Pennink, \& Welvaart, 1987), y por ello es necesario considerarlos como componentes distintos.

Según Shin y Johnson (1978) la satisfacción con la vida es un juicio subjetivo mediante el cual la persona evalúa la calidad de su vida teniendo en cuenta una serie de criterios propios, contra sus estándares. Lucas (2007) comprobó que este proceso se ve influenciado en gran medida por una cuestión de rasgo ya que los resultados mostraron que la disposición de cada persona puede llegar a explicar hasta un $38 \%$ de la varianza. Sin embargo aún queda un componente situacional importante por explicar.

Debido a la importancia que tiene la satisfacción con la vida, se han desarrollado diversas herramientas con el propósito de medir este componente del bienestar subjetivo. Bigot (1974) desarrolló el "Life Satisfaction IndexWell-being" (LSI-W) y Lawton (1975) la Philadelphia Geriatric Center Morale Scale. Estas escalas presentaban algunas limitaciones que Diener, Emmons, Larsen, y Griffin (1985) intentaron resolver creando la 
Satisfaction With Life Scale (SWLS). La escala de satisfacción con la vida es una escala de cinco ítems en formato Likert, que en su conjunto pretenden medir la sensación de satisfacción que tiene un individuo respecto a su vida en cualquier aspecto y momento de esta. La SWLS ha sido traducida y validada en múltiples idiomas como el francés (Blais, Vallerand, Pelletier, \& Briere, 1989), el español (Atienza, Pons, Balaguer \& García-Merita, 2000), el holandés (Arrindell, Heesink \& Feij, 1999), el chino (Sachs, 2004), el hebreo (Anaby, Jarus, \& Zumbo, 2010), el portugués (Gouveia, Milfont, da Fonseca, \& Coelho, 2009; Sancho, Galiana, Gutiérrez, Francisco, y Tomás, 2014), el alemán (Glaesmer, Grande, Braehler, y Roth, 2011) y por supuesto en inglés, el idioma en que se creó originariamente y en el cual se han realizado una gran cantidad de estudios de validación (Diener et al., 1985; Lucas, Diener, \& Suh, 1996; Pavot, Diener, Colvin, \& Sandvick, 1991; Shevlin \& Bunting, 1994). Toda esta evidencia transcultural tiene en común unos resultados que apoyan la idea de una estructura unidimensional, sostenida tanto por análisis factoriales exploratorios como confirmatorios (Diener et al., 1985; Pavot et al., 1991; Sancho et al., 2014; Shevlin, Brunsden, \& Miles, 1998).

La SWLS no solamente se emplea en población general, sino que se ha probado válida para medir la satisfacción con la vida en muestras muy específicas. En este sentido, recientemente se ha estudiado la validez de la SWLS en muestras con enfermedades y problemas de diversa índole. Por ejemplo, un estudio sobre las propiedades psicométricas de esta escala concluyó que es completamente apta para evaluar enfermos de Parkinson (Rosengren, Jonasson, Brogårdh \& Lexell, 2015). Por otro lado, Lucas-Carrasco, Den Oudsten, Eser, y Power (2014) estudiaron este mismo asunto con enfermos de Parkinson de distintos países de Europa, con resultados similares. También se ha validado en un muestra española de enfermos de esclerosis múltiple, con buenos resultados (Lucas-Carrasco et al., 2014). Por último, cabe destacar el estudio de Aishvarya et al. (2014), en el que se concluyó, usando una muestra malasia de 483 personas con distintas enfermedades, tanto físicas como psíquicas, que esta escala es válida y fiable para su uso con personas de estas características.

Los estudios de validación de la SWLS han incluido también el estudio de su invarianza factorial a través de subgrupos dentro de una misma población $y$, también, a través de poblaciones diferentes. Por ejemplo, en un estudio en que se comparaban rusos y estadounidenses, se encontró evidencia de la existencia de invarianza (Tucker, Ozer, Lyubomirsky, \& Boehm, 2006). En el caso de Brasil, la invarianza de medida se probó a través de cinco muestras: estudiantes de bachillerato, estudiantes de grado universitario, profesores de escuela primaria, médicos y población general. En este caso, solamente se halló evidencia de invarianza configural, pero no de invarianza métrica o escalar (Gouveia et al., 2009).

En cuanto a los estudios que se refieren a invarianza entre géneros, los resultados han sido diversos. Existen estudios que han obtenido resultados de invarianza estricta (escalar) en muestras tanto inglesas (Shevlin et al., 1998) como noruegas (Clench-Aas, Nes, Dalgard, \& Aarø, 2011). En esta muestra también se encontró invarianza escalar. Lo mismo ocurrió en una muestra sueca (Hultell \& Gustavson, 2008). Sin embargo, en otra muestra noruega solo se encontró evidencia para invarianza métrica (Moksnes, Løhre, Byrn, \& Haugan, 2014). Otros estudios indican invarianza parcial (Atienza, Balaguer, y GarcíaMerita, 2003). También se ha puesto a prueba si esta escala es igualmente válida en distintos momentos del ciclo vital. Por ejemplo, Atienza et al. (2000) encontraron invarianza en dos muestras españolas de ancianos y estudiantes, 
tanto configural como métrica, al igual que el estudio de la versión alemana realizado por Glaesmer et al. (2011).

Otro punto de interés en la satisfacción con la vida es la variación que puede experimentar a lo largo del ciclo vital. Los resultados que se han encontrado hasta ahora no dejan nada claro este aspecto. En algunos estudios se ha descubierto una relación lineal positiva entre edad y satisfacción con la vida (Hansson, Hillerås, \& Forsell, 2005). En otros la relación hallada ha sido negativa (Chen, 2001). En otros, la relación ha sido curvilínea, lo cual indica que el periodo en el cual la satisfacción con la vida es mayor es hacia la mitad de esta (Easterlin, 2006). Y en otros no se ha encontrado ningún tipo de relación (Diener \& Suh, 1998).

Teniendo en cuenta la importancia de esta escala, parece adecuado realizar una nueva validación de la versión portuguesa de la SWLS. Hay distintos factores que hacen vital dicha validación, como que no existe evidencia de validaciones factoriales en una escala de estas características en población angoleña. Esto significa que este trabajo resulta en una aportación más sobre la evidencia transcultural de la que ya se dispone, aumentando el apoyo a una escala que es considerada un índice excelente de la satisfacción con la vida. Otro interés adicional para realizar este trabajo es ofrecer, por primera vez, evidencia basada en dos modelos psicométricos, la Teoría Clásica de Tests (TCT) que emplea análisis factorial confirmatorio, y la Teoría de la Respuesta al Ítem, en concreto el modelo de respuesta graduada, un modelo que no solamente no se había realizado en una muestra de estas características, sino que aún no se había aplicado a la SWLS.

\section{METODOLOGÍA}

Muestra y procedimiento. La selección de los participantes fue incidental o muestra por conveniencia.
La formaron 1332 jóvenes procedentes de tres provincias del norte, centro y sur de Angola. La edad estaba comprendida entre los 18 y los 30 años ( $M=23,02$; $\mathrm{SD}=3,640)$. El $56,9 \%$ de la muestra está formada por hombres y el $43,1 \%$ por mujeres. Un $42,1 \%$ de la muestra no había trabajado nunca, y del 57'9 \% restante un $21^{\prime} 2 \%$ estaba desempleado en ese momento. Un 36,6\% contaba con un puesto de trabajo.

La muestra fue seleccionada en las tres ciudades más representativas: Luanda, Benguela y Uíge. El criterio principal para la selección de estas ciudades fue porque Luanda, la capital, ha venido recogiendo de manera sucesiva a gran cantidad de la población angoleña procedente de todos los puntos del país (debido a los desplazamientos forzados generados por sucesivas guerras), con lo cual es uno de los lugares más representativos de toda la población. Por otro lado, Benguela, que se encuentra en el sur de Angola, es una de las ciudades más activas en cuanto a creación de empleo, dado su nivel de desarrollo. Por último, Uíge es una de las ciudades del norte de Angola con un buen desarrollo agrícola y en la que están resurgiendo diversas empresas capaces de proporcionar empleo a los jóvenes.

Tras contar con la aprobación del Ministerio de Trabajo y Reinserción Social, se solicitó permiso a diversos centros de empleo, centros de trabajo e instituciones escolares para realizar la investigación. Todos los participantes fueron informados de la naturaleza del estudio y del carácter voluntario de su participación en el mismo, así como de la garantía del anonimato de sus manifestaciones. La aplicación de los cuestionarios fue llevada a cabo, previo entrenamiento para ello, por cinco jóvenes universitarios que participaban en un Máster de Intervención Psicosocial y Desarrollo Humano. Los instrumentos fueron aplicados en centros de empleo, en sus lugares de trabajo, centros educativos, e incluso en la calle. El tiempo dedicado a la respuesta de los cuestionarios fue entre 30 y 45 minutos. 
Medidas. Las encuestas aplicadas a los participantes en este estudio incluían diversas medidas sociodemográficas, así como diversas escalas psicológicas de interés:

La Satisfaction With Life Scale (SWLS; Diener et al., 1985), compuesta de 5 ítems a los que se debía responder en una escala Likert desde (1) totalmente en desacuerdo hasta (5) totalmente de acuerdo. Para esta muestra se obtuvo un alfa de Cronbach de .73, respondiendo a parte del objetivo de validación.

Rosenberg Self-Esteem Scale (RSES). Se presentó a los participantes con cinco alternativas de respuesta, desde (1) totalmente en desacuerdo, hasta (5) totalmente de acuerdo. Para esta muestra se obtuvo un alfa de Cronbach de .65. Tan solo se emplearon los ítems de la escala formulados en positivo, dados los persistentes efectos de método que se han encontrado asociados a los ítems redactados en negativo (Tomás, Oliver, Galiana, Sancho, \& Lila, 2013). Multidimensional Scale of Perceived Social Support (MSPSS; Edwards, 2004). Esta versión evalúa tres dimensiones: Apoyo de la familia, apoyo de los amigos y apoyo de otros significativos. Los participantes debían responder a los 12 ítems con cinco alternativas, desde (1) totalmente en desacuerdo, hasta (5) totalmente de acuerdo. Para esta muestra se obtuvo un alfa de Cronbach de .85 .

Emotional Intelligence Scale (EIS; Wong \& Law, 2002). Esta escala está compuesta por cuatro subescalas, de las cuales, para evaluar el control emocional de los jóvenes estudiados, se empleó la subescala "Control de la emoción". Los participantes contestaron a los cuatro ítems de esta subescala a través de cinco alternativas de respuesta desde (1) totalmente en desacuerdo, hasta (5) totalmente de acuerdo. Para esta muestra se obtuvo un alfa de Cronbach de .83 .
Brief Resilience Scale (BRS; Smith et al., 2008). Esta escala de resiliencia es unidimensional y está compuesta por seis ítems (tres redactados en sentido positivo y tres en negativo). Se eliminaron dos ítems, 1 y 3, por un muy deficiente funcionamiento psicométrico. La consistencia interna para los restantes ítems fue de .61.

Índice de Auto-Percepción de Salud. Este índice es una medida subjetiva de la salud y la calidad de vida recomendado por la OMS. El índice comprende la pregunta: ¿Cómo describiría usted su estado general de salud en este momento? Para responder se ofrecieron cinco alternativas: desde (1) muy mala, hasta, (5) muy buena.

Coping With Job Loss Scale (CWJLS; Latack, 1986). Para medir las estrategias de coping ante el desempleo de los jóvenes angoleños se empleó la versión de Lai y Wong (1998). Está compuesta por 11 ítems que forman dos factores: (1) Búsqueda proactiva de empleo y (2) Distanciamiento de la pérdida del empleo. Se les pidió a los participantes que respondieran a la pregunta: ¿Con qué frecuencia utiliza las siguientes estrategias para superar el desempleo? Las alternativas de respuesta fueron desde (1) casi nunca, hasta (5) casi siempre. Para esta muestra se obtuvo un alfa de Cronbach de .77.

Análisis. Para valorar la validez factorial se realizó un análisis factorial confirmatorio de la SWLS. Para valorar el ajuste de los datos a la estructura factorial esperada (un solo factor), se emplearon diversos índices y estadísticos de ajuste: prueba de chi-cuadrado (Kline 1998; Ullman 1996), el Índice de ajuste comparativo (Comparative Fit Index, CFI), que según Bentler (1990) ha de ser superior a .90, como mínimo, y es excelente por encima de .95; y el Error medio cuadrático de aproximación (Root Mean Squared Error of Aproximation) RMSEA, que ha de ser igual o inferior a .08, y excelente por debajo de .05 (Steiger \& Lind 1980). El 
modelo de análisis factorial confirmatorio se ha estimado en Mplus con estimación WLSMV (Weighted Least Squares Mean and Variance corrected), el más adecuado para los datos provenientes de escalas tipos Likert de cinco anclajes como la de esta escala y ausencia de normalidad multivariante. Este método de estimación solamente permite calcular los índices de ajuste que se han presentado. Para valorar las características psicométricas de la escala y los ítems, también se empleó un modelo de teoría de respuesta al ítem, en concreto, el modelo de respuesta graduada de dos parámetros usando máxima verosimilitud y correcciones robustas.

El ajuste del modelo por TRI fue evaluado mediante pruebas de razón de verosimilitud (LRT) y criterios de información como el Akaike Information Criteria (AIC), el Bayesian Information Criteria (BIC) y el Adjusted Bayesian Criteria (ABIC). Valores bajos de estos criterios indican mejor ajuste del modelo (Raykov \& Marcoulides, 2011).

El modelo de 2 parámetros (2PL), a diferencia del modelo de 1 parámetro (1PL), calcula el índice de discriminación de cada ítem, pues no asume que todos los ítems discriminen lo mismo, por lo tanto, el parámetro (a) indica la pendiente por la cual las respuestas a los ítems cambian en función del nivel de "habilidad" o del nivel del constructo latente que se está midiendo. Estas pendientes normalmente van de 0 a 3 considerándose cualquier valor por encima de 1 como discriminante. Por otra parte, el parámetro (b) indica la dificultad del ítem, en el caso de la SWLS tendremos 4 parámetros (b) por ítem ya que la escala de puntuación va de 1 a 5 .

Por último la precisión de las medidas fue calculada mediante la curva de información del test. Dichas curvas representan la cantidad de información que la escala ofrece en los distintos puntos de la variable latente. los de la estructura y las características psicométricas se calcularon mediante Mplus 6.7 (Muthén \& Muthén, 2007).
Por último se calcularon estimaciones de consistencia interna para ítems y escalas, y se realizaron diversas correlaciones con el objetivo de elaborar una valoración de validez nomológica. Todas estas mediciones se realizaron mediante el paquete estadístico SPSS 22.

\section{RESULTADOS}

Análisis Factorial Confirmatorio. Como hemos señalado previamente, uno de los objetivos del estudio fue estudiar la validez factorial de la SWLS en una muestra de jóvenes angoleños. En base a la literatura se esperaba una solución unifactorial. Los índices de ajuste apoyan esta estructura: $X^{2}(5)=24.03, p<0.001$; CFI $=.99 ;$ y RMSEA $=0.053$. Los índices muestran un ajuste adecuado. Por otro lado, las saturaciones factoriales también apoyaron el modelo propuesto, pues todas fueron estadísticamente significativas $(p<0.001)$, abogando por el modelo unifactorial y elevadas, pues oscilaron entre un mínimo de .484 (ítem 1) hasta un máximo de .760 (ítem 2).

Modelo de respuesta graduada. EI modelo de dos parámetros presenta el siguiente ajuste: LRT (3092) $=2699.241$, $p=1$, AIC $=18986.0, \mathrm{BIC}=19115.8, \mathrm{y}$ ABIC $=19036.4$. En la tabla 1 podemos ver cómo los parámetros de discriminación de todos los ítems son superiores a 1 , lo que indica una alta discriminación. También muestra que su ordenación es correcta, y están en los rangos adecuados.

La figura 1 ofrece las curvas características de cada uno de los ítems de la escala. Estas curvas muestran de forma gráfica los parámetros $a$ y $b$ que se muestran en la tabla 1 . Si la curva tiene una pendiente muy apuntada es que discrimina muy bien, pero en un espacio del rasgo relativamente pequeño (parámetro a). Los parámetros b (sumados) ofrecen la localización de la máxima información del ítem en la escala. Por su parte, la figura 2 viene a representar al test en su conjunto. Se suma la cantidad de información que ofrecen los cinco ítems y con ella se dibuja la función de información del 
Tabla 1. Estadísticos descriptivos.

\begin{tabular}{lcccc}
\hline \multicolumn{1}{c}{ Variables } & Media & DT & \multicolumn{2}{c}{ Asimetría } \\
\hline Auto-Estima & 3.91 & 0.67 & -0.76 & 0.92 \\
Apoyo Familiar & 3.79 & 0.93 & -0.91 & 0.41 \\
Apoyo de amigos & 3.51 & 0.91 & -0.55 & -0.12 \\
Apoyo de otros & 4.04 & 0.64 & -1.13 & 1.06 \\
Satisfacción con la vida & 2.85 & 0.85 & 0.61 & -0.34 \\
Control Emocional & 3.71 & 0.86 & -0.67 & 0.22 \\
Resiliencia & 3.03 & 0.78 & 0.03 & -0.28 \\
Salud Mental & 3.09 & 0.64 & -0.20 & 0.25 \\
Búsqueda proactiva de empleo & 3.25 & 1.34 & -0.30 & -1.2 \\
Distanciamiento de la pérdida & 3.18 & 0.95 & -0.04 & -0.43 \\
del empleo & 3.39 & 0.99 & 0.03 & -0.02 \\
Estado de Salud & & & & \\
\hline
\end{tabular}

test. Esto es, la información sobre la fiabilidad (función de información) de la escala se muestra en la figura 2. Dado que la escala del rasgo es en valores tipificados, al observar tanto la figura 1 , como, sobre todo, la figura 2, se puede ver que la máxima información se obtiene en los valores promedios de la satisfacción con la vida.

Consistencia interna. Se obtuvo un resultado de 0.73 al calcular el a de Cronbach. En la tabla 2 podemos ver cómo la homogeneidad de los ítems va de .392 a .578 , por lo que se puede considerar adecuada. Los ítems se encuentran alta y homogéneamente correlacionados. Por último, el índice de consistencia no mejoraría quitando

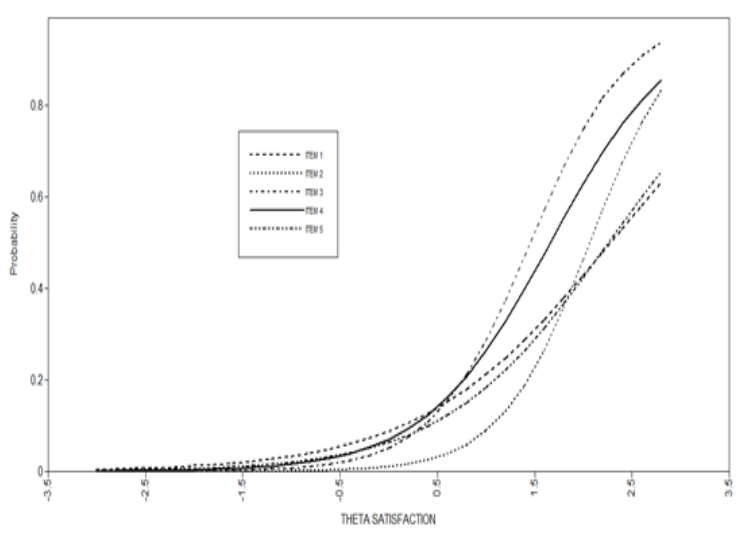

Figura 1. Curvas características de los cinco ítems de la SWLS: parámetros de discriminación (a) y localización (b).
Tabla 2. Parámetros de discriminación (a) y dificultad (b) para todos los ítems del SWLS.

\begin{tabular}{cccccc}
\hline Item & $\mathbf{a}$ & $\mathbf{b}_{\mathbf{1}}$ & $\mathbf{b}_{\mathbf{2}}$ & $\mathbf{b}_{\mathbf{3}}$ & $\mathbf{b}_{\mathbf{4}}$ \\
\hline 1 & 1.02 & -2.75 & -1.29 & -0.03 & 2.34 \\
2 & 2.18 & -2.35 & 0.10 & 2.31 & 4.50 \\
3 & 1.99 & -2.84 & -0.74 & 0.85 & 2.90 \\
4 & 1.55 & -2.34 & -0.37 & 0.82 & 2.58 \\
5 & 1.18 & -1.22 & 0.32 & 1.49 & 2.68 \\
\hline
\end{tabular}

cualquiera de los ítems, por lo que podríamos concluir que la consistencia interna de la escala y sus ítems es adecuada.

Validez nomológica. Con el objetivo de analizar la validez nomológica del instrumento, se relaciona el factor de "satisfacción con la vida" con distintas variables con las que es esperable que se mantengan relaciones significativas, tal como ha venido mostrando la literatura científica. Se esperaban relaciones positivas con el apoyo social, con la autoestima, la salud física y mental percibida, y la resiliencia. Con respecto a las relaciones que se esperaban encontrar entre satisfacción vital y el afrontamiento, se esperaba una relación positiva con la búsqueda activa de empleo y una negativa con el distanciamiento (afrontamiento pasivo). El apoyo social percibido correlacionó de forma positiva,

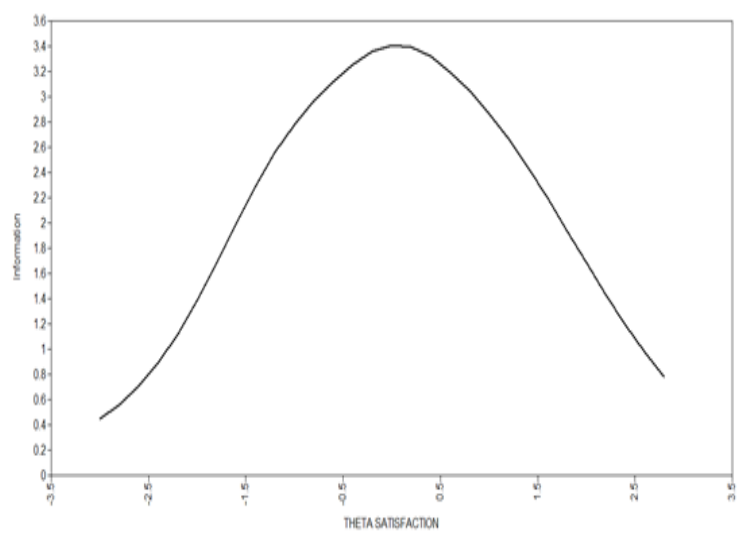

Figura 2. Función de información del test: suma de la cantidad de información individual ofrecida por cada ítem. 
Gutiérrez et al - Propiedades psicométricas de la satisfaction with life scale en jóvenes angoleños

Tabla 3. Medias, desviaciones típicas (DT), correlación ítem-total $\left(r_{i t}\right)$, alfa de la escala si se elimina el ítem y correlaciones entre los ítems.

\begin{tabular}{|c|c|c|c|c|c|c|c|c|}
\hline \multirow{2}{*}{ Item } & \multirow{2}{*}{ Media } & \multirow{2}{*}{ DT } & \multirow[b]{2}{*}{$r_{i t}$} & \multirow{2}{*}{ a sin el item } & \multicolumn{4}{|c|}{ Correlaciones entre ítems } \\
\hline & & & & & 1 & 2 & 3 & 4 \\
\hline 1 & 3.31 & 1.13 & .39 & .72 & - & & & \\
\hline 2 & 2.54 & 1.11 & .57 & .66 & .30 & - & & \\
\hline 3 & 3.00 & 1.25 & .56 & .66 & .31 & .52 & - & \\
\hline 4 & 2.92 & 1.28 & .52 & .67 & .30 & .43 & .41 & - \\
\hline 5 & 2.49 & 1.28 & .43 & .71 & .22 & .35 & .32 & .35 \\
\hline
\end{tabular}

obteniendo la dimensión del apoyo familiar la correlación más alta $(r=0.311$, $\mathrm{p}<0.01)$, el apoyo de otros significativos mostró un valor de .283 $(p<0.01)$ y el apoyo de los amigos una correlación de $0.281(p<0.01)$. También se encontró relación positiva con la autoestima $(0.288, p<0.01)$. El control emocional también correlacionó positivamente con la satisfacción con la vida $(0.238, \mathrm{p}<0.01)$, así como la percepción de salud $(0.215$, $\mathrm{p}<0.01)$. También correlacionaron positivamente, aunque con menor valor, la resiliencia $(0.169, \mathrm{p}<0.01)$, la salud mental $(0.115, \mathrm{p}<0.01)$ y por último, la búsqueda activa de empleo, (0.078, $\mathrm{p}<0.05$ ).

\section{DISCUSIÓN}

El objetivo de esta investigación era aportar nueva evidencia sobre las propiedades psicométricas de la SWLS, no solo desde la TCT, sino también desde la TRI, algo muy novedoso ya que no hay evidencia psicométrica con esta escala desde los modelos de TRI, intentando de esta manera aumentar el apoyo a dicha escala. El modelo de respuesta graduada aplicado se combina con los resultados de la TCT, y aporta ventajas sobre esta. En concreto, y para nuestros objetivos, ofrece estimaciones de los parámetros de los ítems invariantes de muestra a muestra, y adicionalmente mediante la curva de información del test nos ofrece información de la fiabilidad de la escala a través de los distintos niveles de satisfacción, en lugar de una estimación puntual promedio para cualquier nivel del constructo medido (Zickar \& Broadfoot, 2009).

El constructo "satisfacción con la vida" es clave para la psicología y sus diversas ramas. Pero sobre todo es muy relevante para todo ser humano. Según Diener y Emmons (1984) dicho constructo se halla implícito en el de bienestar subjetivo, entendiéndose este como la sensación que experimenta una persona provocada por todo aquello que le haga sentir bien en relación con sus propios valores (Diener et al., 2003).

La mayoría de investigaciones sobre la SWLS se han centrado en estudiar sus propiedades psicométricas como validez o fiabilidad, siempre desde una perspectiva de la TCT (Anaby et al., 2010; Arrindell et al., 1999; Atienza et al., 2000; Blais et al., 1989; Diener et al., 1985; Gouveia et al., 2009; Sachs, 2004; Sancho et al., 2014; Glaesmer et al., 2011; Lucas et al., 1996; Pavot et al., 1991; Shevlin y Bunting, 1994). En este trabajo se decidió verificar y ampliar los estudios previos con la intención de generar más evidencia respecto a las propiedades psicométricas de esta escala, ya que en algunos casos, como en el de la validez factorial, era más bien escasa. Pero también se trató de aportar información sobre aspectos que han recibido menos atención como es estimar el modelo de respuesta graduada, un modelo de TRI que ofrece nueva información.

En cuanto a los resultados de validez factorial, la evidencia muestra claramente que una única dimensión de satisfacción 
ajusta bien a los datos observados. Este es un resultado que concuerda con los obtenidos en otras investigaciones realizadas sobre la versión portuguesa de la SWLS (Gouveia et al., 2009; Sancho et al., 2014). Este hallazgo no se ha encontrado tan solo en la versión portuguesa, sino que es un resultado bien establecido a través de distintas lenguas (Blais et al., 1989; Atienza et al., 2000; Arrindell et al., 1999; Sachs, 2004; Anaby et al., 2010) por lo que parece comprensible asumir que actualmente esta es la solución que mejor ajusta de manera general.

En cuanto a los cálculos de TRI, hay que tener en cuenta que no existen trabajos que hayan aplicado el modelo de respuesta graduada sobre los datos de esta escala, por lo que la comparación no es posible. Se calculó un modelo de dos parámetros que ajustó adecuadamente, por lo que se podría afirmar que los resultados desde TRI refuerzan la idea que ya desde la TCT se tenía de la escala: una escala unidimensional y fiable para medir satisfacción con la vida. Se estimaron las CCI de los ítems, obteniendo también buenos resultados, pues todos los ítems resultaron ser muy discriminantes, sobre todo los ítems 2,3 y 4 en los cuales se obtuvo un parámetro de discriminación superior a 1.5 .

Respecto a la fiabilidad, los análisis proporcionaron evidencia de niveles adecuados de consistencia interna de la SWLS en esta muestra. Cierto es que en ocasiones se ha informado de niveles de consistencia interna superiores al obtenido en este estudio (Diener et al., 1985) pero eso es debido probablemente a las características de la muestra, por lo que se sigue considerando como adecuado este estudio. Con la teoría de respuesta al ítem se ha obtenido una información muy valiosa sobre la fiabilidad a lo largo del continuo de satisfacción, y se ha visto que la escala es altamente fiable en valores promedio y alrededores de satisfacción. Esto es, es muy adecuada para población general.
Por último, la validez nomológica indicó que la evaluación de la SWLS es consistente con medidas externas que, en teoría, deberían estar correlacionadas con ésta, pues la satisfacción con la vida correlacionó positivamente con todas las medidas de un modo significativo. Tan solo obtuvimos una correlación no significativa en distanciamiento dela búsqueda de empleo, uno de los tipos de coping por evitación que, efectivamente debiera estar menos relacionado con la satisfacción vital. Viendo que las medidas con las que correlacionó, se esperaba que se relacionaran positivamente con satisfacción con la vida, pensamos que los resultados apoyan la idea de que la SWLS es un instrumento válido desde el punto de vista nomológico.

Es importante tener en cuenta las distintas limitaciones que puede presentar el estudio. El principal objetivo del estudio era validar la SWLS. A pesar de haber realizado análisis de validez factorial y validez nomológica se han obviado otros tipos de validez, como la concurrente o la convergente. Por eso mismo, sería conveniente medir los constructos psicológicos necesarios para realizar este tipo de validaciones en próximas investigaciones, lo cual aumentaría la información disponible sobre las propiedades psicométricas de esta escala.

\section{CONCLUSIONES}

En conclusión, este estudio aporta de nuevo evidencia a favor de las adecuadas propiedades psicométricas de esta escala para medir la satisfacción con la vida, en este caso en jóvenes angoleños y con la versión de la escala en portugués. A su vez, ofrece información más novedosa sobre algunas de sus características, por lo cual, estudios futuros permitirán contrastar para determinar con un menor margen de error su potencial aplicabilidad. 
Gutiérrez et al - Propiedades psicométricas de la satisfaction with life scale en jóvenes angoleños

\section{REFERENCIAS}

Aishvarya, S., Maniam, T., Karuthan, C., Sidi, H., Jaafar, N., \& Oei, T. (2014). Psychometric properties and validation of the Satisfaction with life scale in psychiatric and medical outpatients in Malaysia. Comprehensive Psychiatry, 55, 101-106.

Anaby, D., Jarus, T., \& Zumbo, B. D. (2010). Psychometric evaluation of the hebrew language version of the satisfaction with life scale. Social Indicators Research, 96, 267-274.

Andrews, F. M. (1974). Social indicators of perceived life quality. Social Indicators Research, 1, 279-299.

Andrews, F. M., \& Withey, S. B. (1976). Social indicators of well-being: America's perception of life quality. New York: Plenum.

Arrindell, W. A., Heesink, J., \& Feij, J. A. (1999). The satisfaction with life scale (SWLS): Appraisal with 1700 health young adults in the Netherlands. Personality and Individual Differences, 26, 815-826.

Atienza, F. L., Balaguer, I., \& GarcíaMerita, M. L. (2003). Satisfaction with life scale: analysis of factorial invariance across sexes. Personality and Individual Diferences, 35, 1255-1260.

Atienza, F. L., Pons, D., Balaguer, I., \& García-Merita, M. L. (2000). Psychometric properties of satisfaction with life scale in adolescents. Psicothema, 12, 331-336.

Beiser, M. (1974). Components and correlates of mental well-being. Journal of Health and Social Behavior, 15, 320-327.

Bentler, P. M. (1990). Comparative fit indices in structural models. Psychological Bulletin, 107, 238-246.
Bigot, A. (1974). The relevance of american life satisfaction indices for research on british subjects before and after retirement. Age and Ageing, 2, 113121.

Blais, M. R., Vallerand, R. J., Pelletier, L. G., \& Briere, N. M. (1989). The satisfaction with life scale: cannadianfrench validation of the satisfaction with life scale. Canadian Journal of Behavioral Science, 21, 210-223.

Böckerman, P., Johanson, E., \& Saarni, S.I. (2012). Institutionalisation and subjective wellbeing for old age individuals: is life really miserable in care homes? Ageing and Society, 32, 1176-1192.

Chen, C. (2001). Aging and life satisfaction. Social Indicators Research, 54, 57-79.

Clench-Aas, J., Nes, R. B., Dalgard, O. D., \& Aarø, L. E. (2011). Dimensionality and measurement invariance in the satisfaction with life scale in Norway. Quality of Life Research, 20, 1307-1317.

DeHaes, J. C., Pennink, B. J. W., \& Welvaart, K. (1987). The distinction between affect and cognition. Social Indicators Research, 19, 367-378.

Diener, E. (1984). Subjective well-being. Psychological Bulletin, 95, 542-575.

Diener, E., \& Emmons, R. A. (1984). The independence of positive and negative affect. Journal of Personality and Social Psychology, 47, 1105-1117.

Diener, E., \& Suh, E. M. (1998). Subjective well-being and age: An international analysis. Annual Review of Gerontology and Geriatrics, 17, 304-324.

Diener, E., Emmons, R. A., Larsen, R. J., \& Griffin, S. (1985). The satisfaction with life scale. Journal of Personality Assessment, 49, 71-75. 
Diener, E., Oishi, S., \& Lucas, R. E. (2003). Personality, culture, and subjective wellbeing: Emotional and cognitive evaluations of life. Annual Review of Psychology, 54, 403-425.

Easterlin, R. (2006). Life cycle happiness and its sources: Intersections of psychology, economics, and demography. Journal of Economic Psychology, 27, 463482.

Eckersley, R. (2013). Subjective wellbeing: Telling only half the story. A commentary on Diener et al. (2012). Theory and validity of life satisfaction scales. Social Indicators Research, 112, 529-534.

Edwards, L. (2004). Measuring perceived social support in mexican american youth: Psychometric properties of the Multidimensional Scale of Perceived Social Support. Hispanic Journal of Behavioral Sciences, 26, 187-194.

Glaesmer, H., Grande, G., Braehler, E., \& Roth, M. (2011). The german version of the satisfaction with life scale (SWLS). European Journal of Psychological Assessment, 27, 127-132.

Gouveia, V. V., Milfont, T. L., da Fonseca, P. N., \& Coelho, J. (2009). Life satisfaction in Brazil: Testing the psychometric properties of the satisfaction with life scale (SWLS) in five bazilian samples. Social Indicators Research, 90, 267-277.

Hansson, A., Hillerås, P., \& Forsell, Y. (2005). Well-being in an adult swedish population. Social Indicators Research, 74, 313-325.

Hultell, D., \& Gustavson, J. P. (2008). A psychometric evaluation of the satisfaction with life scale in a swedish nationwide sample of university students. Personality and Individual Differences, 44, 10701079.

Kline, R. B. (1998). Principles and practice of structural equation modeling. New York: Guilford.
Lai, J., \& Wong, W. (1998). Optimism and coping with unemployment among Hong Kong chinese women. Journal of Research in Personality, 32, 454-479.

Latack, J. C. (1986). Coping with job stress: Measures and future directions for scale development. Journal of Applied Psychology, 71, 377-385.

Lawton, M. P. (1975). The Philadelphia center morale scale, a revision. Journal of Gerontology, 30, 85-89.

Lucas, R. E. (2007). How stable is happiness? Using the STARTS model to estimate the stability of life satisfaction. Journal of Research in Personality, 41, 1091-1098.

Lucas, R. E., Diener, E., \& Sub, E. (1996). Discriminant validity of well-being measures. Journal of Personality and Social Psychology, 71, 616 -628.

Lucas-Carrasco, R., Den Oudsten, B., Eser, E., \& Power, M. (2014). Using the satisfaction with life scale in people with Parkinson's disease: A validation study in different european countries. The Scientific World Journal, 2014, 1-7.

Moksnes, U. K., Løhre, A., Byrn, D. G., \& Haugan, G. (2014). Satisfaction with life scale in adolescents: Evaluation of factor structure and gender invariance in a norwegian sample. Social Indicators Research, 118, 657-671.

Monk-Turner, E. \& Turner, C. (2012). Subjective wellbeing in a southwestern province in China. Journal of Happiness Studies, 13, 357-369.

Muthén, L. K., \& Muthén, B. O. (2007). Mplus User's Guide (Sixth Edition). Los Angeles, CA: Muthén \& Muthén.

Oswald, A. J., \& Wu, S. (2010). Objective confirmation of subjective measures of human well-being: Evidence from the USA. Science, 327, 576-579. 
Gutiérrez et al - Propiedades psicométricas de la satisfaction with life scale en jóvenes angoleños

Pavot, W., \& Diener, E. (1993). Review of the satisfaction with life scale. Psychological Assessment, 5, 164-172.

Pavot, W., Diener, E., Colvin, C. R., \& Sandvick, E. (1991). Further validation of the satisfaction with life scale: Evidence for the cross-method convergence of wellbeing. Social Indicators Research, 28, $1-20$.

Raykov, T., \& Marcoulides, G. A. (2011). Introduction to psychometric theory. NY: Routledge.

Renshaw, T. L., Long, A. C. J., \& Cook, C. R. (2015). Assessing teachers' positive psychological functioning at work: Development and validation of the teacher subjective wellbeing questionnaire. School Psychology Quarterly, 30(2), 289-306.

Rosengren, L., Jonasson, S., Brogårdh, C., \& Lexell, J. (2015). Psychometric properties of the satisfaction with life scale in Parkinson's disease. Acta Neurologica Scandinavica, 132, 164-170.

Sachs, J. (2004). Validation of the satisfaction with life scale in a sample of Hong Kong University. Psychologia: An International Journal of Psychology in the Orient, 46, 225-234.

Sancho, P., Galiana, L., Gutiérrez, M., Francisco, E., \& Tomás, J. M. (2014). Validating the portuguese version of the satisfaction with life scale in an elderly sample. Social Indicators Research, 115, 457-466.

Shevlin, M. E., \& Bunting, B. P. (1994). Confirmatory factor analysis of the satisfaction with life scale. Perceptual and Motor Skills, 79, 1316-1318.

Shevlin, M., Brunsden, V., \& Miles, J. N. V. (1998). Satisfaction with life scale: Analysis of factorial invariance, mean structures and reliability. Personality and Individual Differences, 25, 911-916.
Shin, D. C., \& Johnson, D. M. (1978). A vowed happiness as an overall assessment of the quality of life. Social Indicators Research, 5, 475-492.

Smith, B.W., Dalen, J., Wiggins, K., Tooley, E., Christopher, P. y Bernard, J. (2008). The Brief Resilience Scale: Assessing the ability to bounce back. International Journal of Behavioral Medicine, 15, 194200.

Steiger, J. H., \& Lind, C. (1980). Statistically based tests for the number of common factors. Paper presented at the annual meeting of the Psychometric Society, Iowa City, IA

Tomás, J., Oliver, A., Galiana, L., Sancho, P., \& Lila, M. (2013). Explaining method effects associated with negatively worded items in trait and state global and domainspecific self-esteem scales. Structural Equation Modeling: A Multidisciplinary Journal, 20, 299-313.

Tucker, K. L., Ozer, D. J., Lyubomirsky, S., \& Boehm, J. K. (2006). Testing for measurement invariance in the satisfaction with life scale: A comparison of russians and north americans. Social Indicators Research, 78, 341-360.

Ullman, J. B. (1996). Structural equation modeling. In B. Tabachnick \& L. Fidell (Eds.), Using multivariate statistics (pp. 709-812). New York: Harper Collins.

Wong, C. S., \& Law, K. S. (2002). The effects of leader and follower emotional intelligence on performance and attitude: An exploratory study. Leadership Quarterly, 13, 243-274.

Zickar, M. J., \& Broadfoot, A. A. (2009). The partial revival of a dead horse? Comparing Classical Test Theory and Item Response Theory. In C. E. Lance and R. J Vandenberg (Eds.), Statistical and methodological myths and urban legends. New York, NY: Routledge. 